\title{
Marked Truncal Acne Response to Sarecycline Monotherapy in Different Ethnicities
}

Angela Yen Moore ${ }^{1,2}$, Stephen Moore ${ }^{1,3}$, Luke Moore ${ }^{1}$, Ayman Grada ${ }^{4}$, Stephen K Tyring ${ }^{3}$

'Arlington Research Center, Arlington, TX, USA; ${ }^{2}$ Baylor University Medical Center, Dallas, TX, USA

3Department of Dermatology, The University of Texas McGovern Medical School, Houston, TX, USA; 4 Grada Dermatology Research LLC, Chesterbrook, PA, USA

\section{INTRODUCTION}

- $60-70 \%$ of patients present with acne on the trunk. ${ }^{1}$

Since severity of truncal acne does not correlate with severity of facial acne, the psychosocial burden and physical pain and bleeding of truncal acne cannot be underestimated. ?

Oral antibiotics are the mainstay treatment for moderate-to-severe acne vulgaris.

Sarecycline, a narrow-spectrum tetracycline-class drug, is FDA-approved for moderate-to-severe acne vulgaris in patients 9 years of age and older.

Sarecycline exhibits potent anti-inflammatory activity and activity against $C$. acnes but reduced activity against Gram-negative bacteria commonly found in the gut; these characteristics may contribute to its low potential for antibiotic resistance and favorable safety and tolerability profile. ${ }^{3}$

Significant improvement in truncal acne, using IGA assessments, was reported with sarecycline in Phase 3 clinical trials. ${ }^{4,5}$

\section{METHODS}

- We present 2 patients treated with sarecycline monotherapy for 3 months.

- At baseline, weeks 3, 6, 9 and 12, we assessed IGA scores and lesion counts change of the trunk.

\begin{tabular}{|l|l|l|l|l|}
\hline \multicolumn{5}{|c|}{ Baseline Data } \\
\hline Demographics & $\begin{array}{l}\text { Skin } \\
\text { Phototype }\end{array}$ & $\begin{array}{l}\text { Truncal } \\
\text { IGA }\end{array}$ & $\begin{array}{l}\text { Truncal } \\
\text { lesion } \\
\text { count }\end{array}$ & $\begin{array}{l}\text { Facial } \\
\text { lesion } \\
\text { count }\end{array}$ \\
\hline $\begin{array}{l}14 \text { yo White } \\
\text { male }\end{array}$ & 2 & 4 & 47 & 8 \\
\hline $\begin{array}{l}13 \text { yo } \\
\text { Hispanic } \\
\text { female }\end{array}$ & 4 & 3 & 29 & 28 \\
\hline
\end{tabular}

* Inflammatory lesions (papules and pustules)

\section{4-year-old White Male}
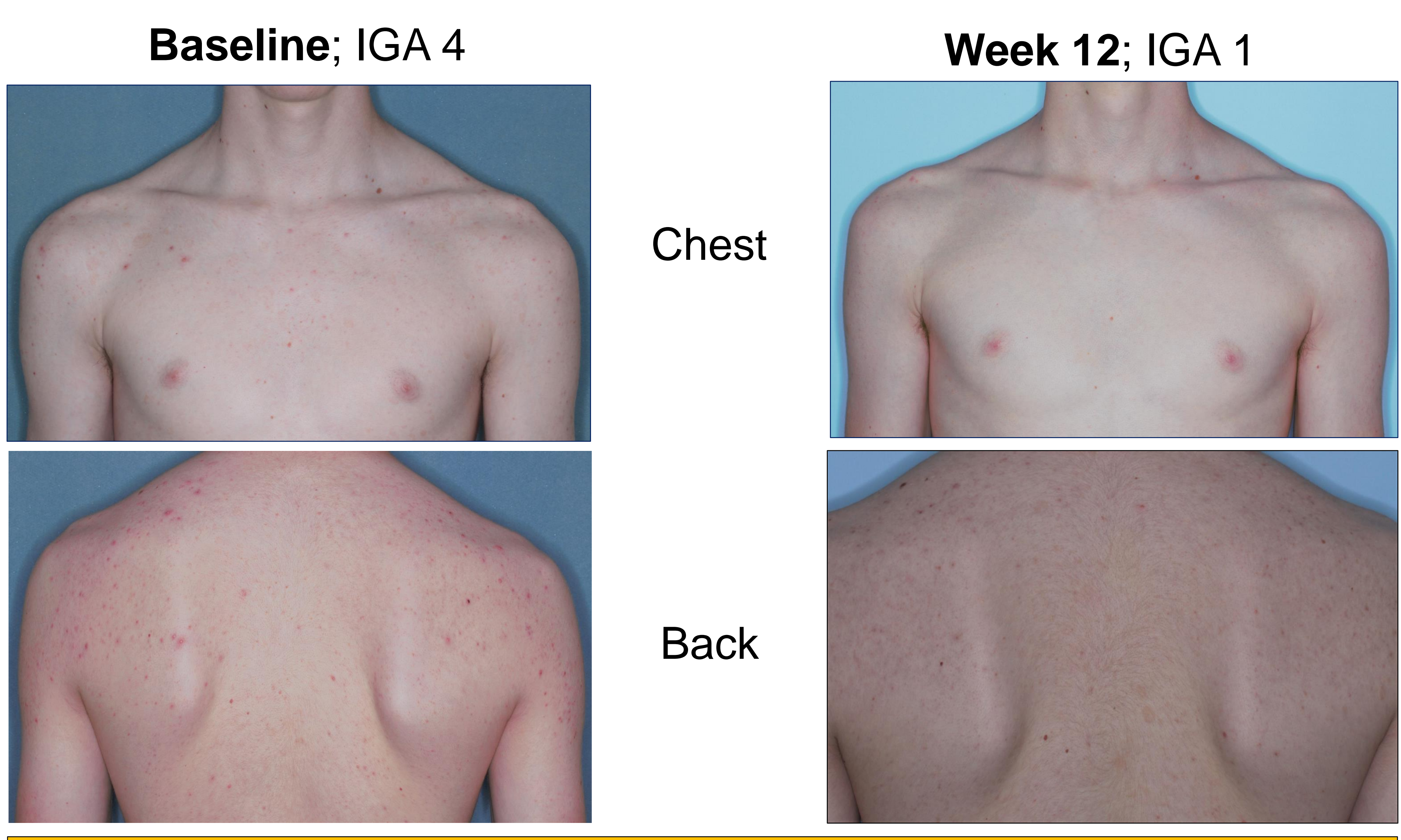

13-year-old Hispanic Female Baseline; IGA 3

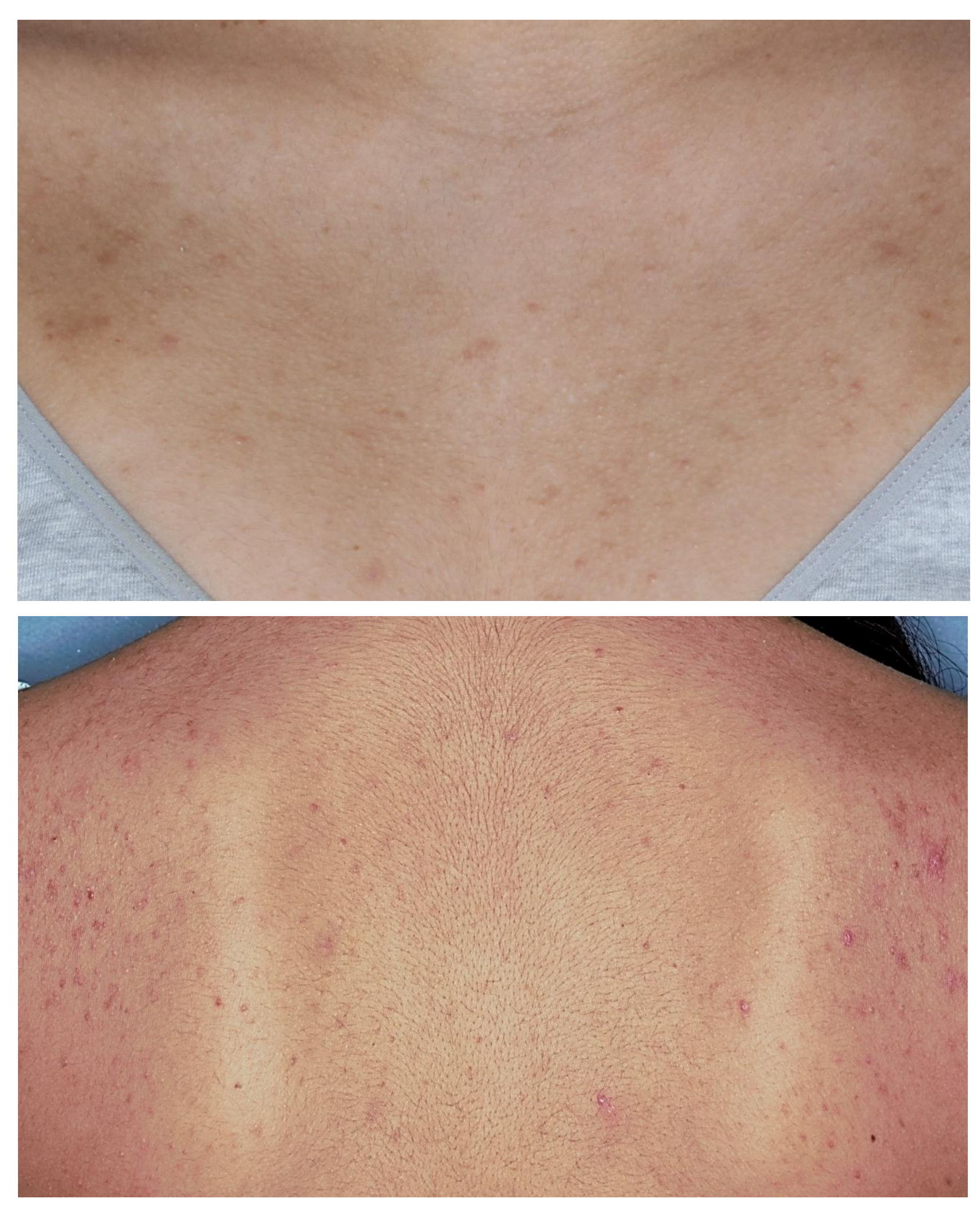

Chest

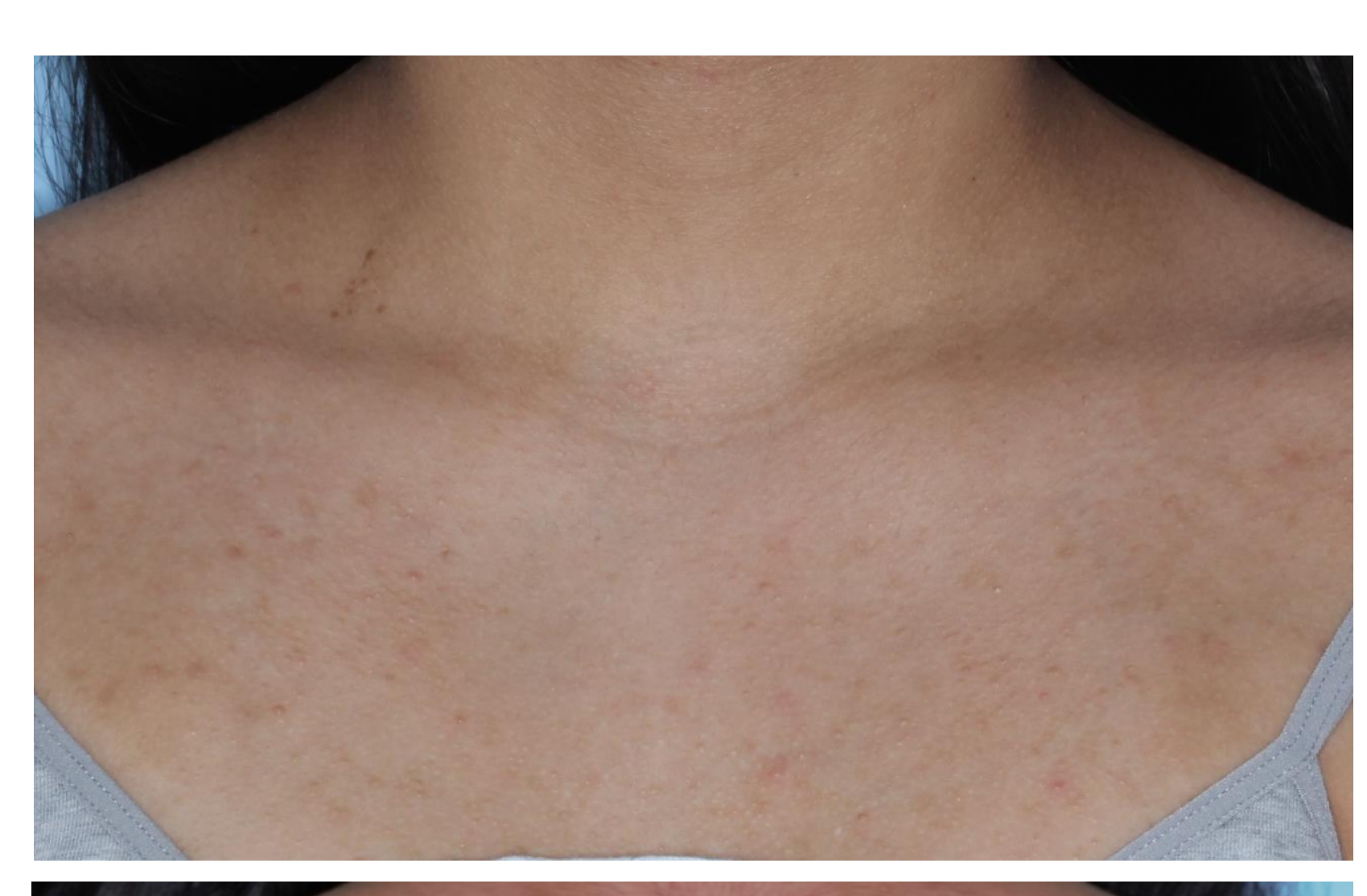

Back

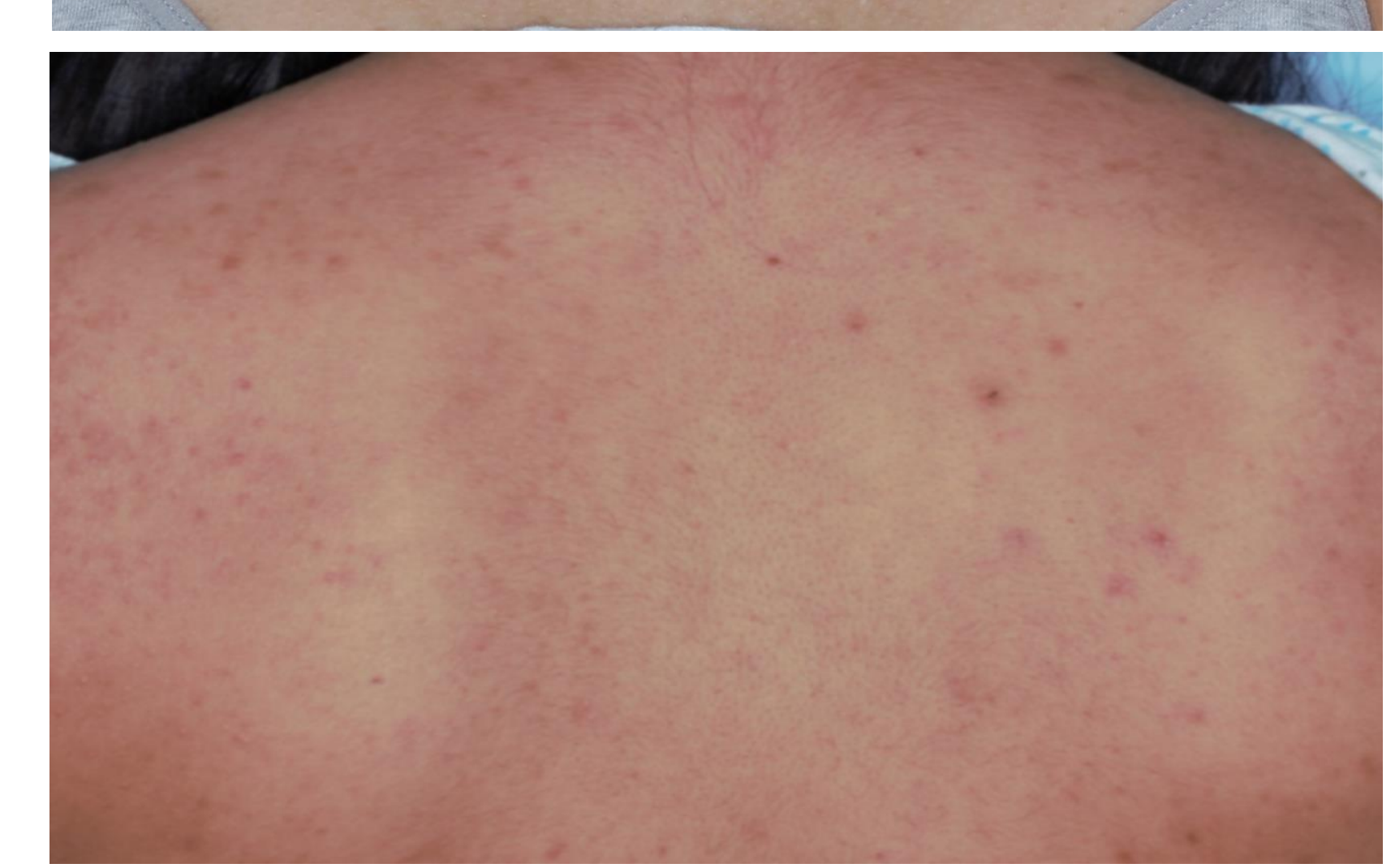

\section{RESULTS}

Inflammatory Lesion Count Reduction

\begin{tabular}{|l|l|l|l|l|l|}
\hline Demographics & Baseline & $\begin{array}{l}3 \\
\text { Wks }\end{array}$ & $\begin{array}{l}6 \\
\text { Wks }\end{array}$ & $\begin{array}{l}9 \\
\text { Wks }\end{array}$ & $\begin{array}{l}12 \\
\text { Wks }\end{array}$ \\
\hline $\begin{array}{l}14 \text { yo White } \\
\text { male }\end{array}$ & 47 & $\begin{array}{l}22 \\
(53 \%)\end{array}$ & $\begin{array}{l}8 \\
(83 \%)\end{array}$ & $\begin{array}{l}1 \\
(98 \%)\end{array}$ & $\begin{array}{l}2 \\
(96 \%)\end{array}$ \\
\hline $\begin{array}{l}13 \text { yo Hispanic } \\
\text { female }\end{array}$ & 29 & $\begin{array}{l}16 \\
(45 \%)\end{array}$ & $\begin{array}{l}4 \\
(86 \%)\end{array}$ & $\begin{array}{l}2 \\
(93 \%)\end{array}$ & $\begin{array}{l}3 \\
(90 \%)\end{array}$ \\
\hline
\end{tabular}

- No side effects of special interest for tetracycline-family antibiotics, including nausea, diarrhea, esophagitis, pseudotumor cerebri, blurry or double vision, dizziness vertigo, or blue-gray pigmentation, were observed.

\section{CONCLUSIONS}

This case series shows rapid improvement in IGA and reduction inflammatory lesion count with oral sarecycline, and confirms previously reported significant improvement of truncal acne in Phase 3 clinical trials

\section{CLINICAL IMPLICATIONS}

- Sarecycline is an effective narrow-spectrum antibiotic alternative that can be utilized in treating truncal acne especially when topical agents are challenging for the patients to apply due to surface area and location

\section{REFERENCES}

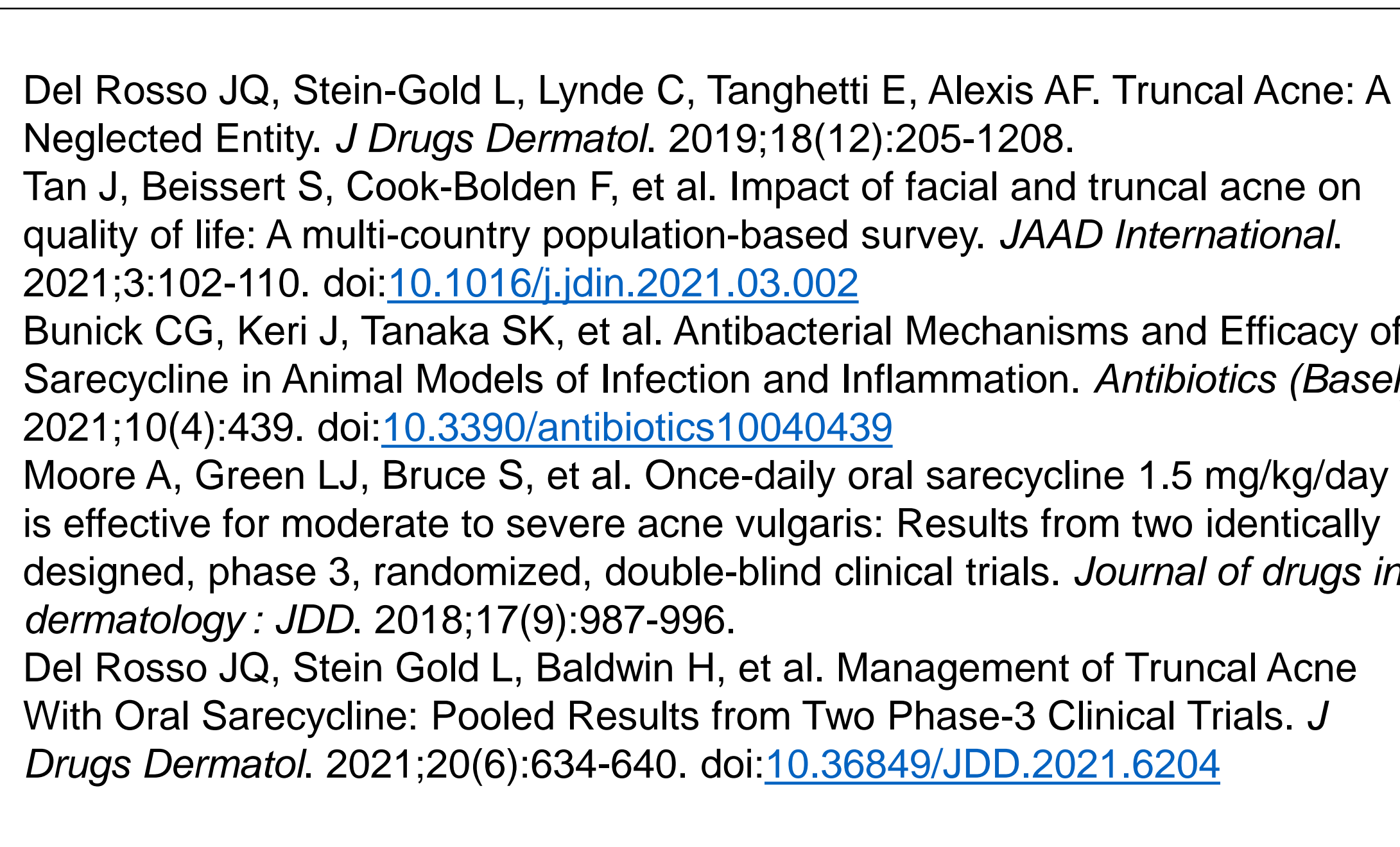

Disclosures: Research grant from Almirall, LLC. 